\title{
Portable Analyzer Based on Microfluidics/Nanoengineered Electrochemical Sensors for In-situ Characterization of Mixed Wastes
}

(First Year of Funding: FY 2004)

\author{
Principal Investigator: \\ Dr. Yuehe Lin \\ Phone: (509) 376-0529 \\ FAX: (509) 376-5106 \\ Email: yuehe.lin@pnl.gov \\ Pacific Northwest National Laboratory \\ P.O. Box 999, MISN K8-93 \\ Battelle Blvd, Richland, WA 99352
}

PNNL Co-Investigators:

Dr. Glen E. Fryxell

Dr. Wassana Yantasee

Dr. Zheming Wang

External Collaborator:

Prof. Joseph Wang

Phone (505) 646-2140

FAX: (505) 646-6033

Email: joewang@nmsu.edu

Department of Chemistry and Biochemistry

New Mexico State University, MSC 3C

Las Cruces, NM 88003 


\section{Research Objective}

Required characterizations of the DOE's transuranic (TRU) and mixed wastes (MW) before disposing and treatment of the wastes are currently costly and have lengthy turnaround. Research toward developing faster and more sensitive characterization and analysis tools to reduce costs and accelerate throughputs is therefore desirable. This project is aimed at the development of electrochemical sensors, specific to toxic transition metals, uranium, and technetium, that can be integrated into the portable sensor systems. This system development will include fabrication and performance evaluation of electrodes as well as understanding of electrochemically active sites on the electrodes specifically designed for toxic metals, uranium and technetium detection. Subsequently, these advanced measurement units will be incorporated into a microfluidic prototype specifically designed and fabricated for field-deployable characterizations of such species. The electrochemical sensors being investigated are based on a new class of nanoengineered sorbents, Self-Assembled Monolayer on Mesoporous Supports (SAMMS). SAMMS are highly efficient sorbents due to their interfacial chemistry that can be fine-tuned to selectively sequester a specific target species. Adsorptive stripping voltammetry (AdSV) will be performed on two classes of electrodes: the SAMMS modified carbon paste electrodes, and the SAMMS thin film immobilized on microelectrode arrays. Interfacial chemistry and electrochemistry of metal species on the surfaces of SAMMS-based electrodes will be studied. This fundamental knowledge is required for predicting how the sensors will perform in the real wastes which consist of many interferences/ligands and a spectrum of $\mathrm{pH}$ levels. The best electrode for each specific waste constituent will be integrated onto the portable microfluidic platform. Efforts will also be focused on testing the portable microfluidics/electrochemical sensor systems with the selected MW and TRU waste samples at the Hanford site. The outcome of this project will lead to the development of a portable analytical system for in-situ characterization of MW and TRU wastes. The technology will greatly reduce costs and accelerate throughputs for characterizations of MW and TRU wastes.

\section{Research Progress and Implications}

This report summarizes work after 1 year of a 3 year project.

\section{Synthesis and evaluation of SAMMS}

Both heavy-metal selective and actinide-selective SAMMS materials have been synthesized and characterized. The binding kinetics, $\mathrm{K}_{\mathrm{d}}$, and loading capacity of phosphonic acid SAMMS and hydroxyphosphonamide (HOPO) SAMMS for heavy metals, lanthanides, and actinides have been evaluated. Results indicated that SAMMSs can rapidly and effectively adsorb heavy metal, lanthanides, and actinide ions from aqueous solution. Detailed information is available from the recent publications generated from this project (see publications part).

\section{Nanoengineered electrochemical sensors based on SAMMS}

Two classes of sensors are investigated: the SAMMS modified carbon paste electrodes, and the SAMMS thin-film as a sensing layer on microchip electrodes. We have found very encouraging results as discussed below.

\subsection{Electrochemical sensors based on chemically modified carbon paste electrodes.}

Electrodes for adsorptive stripping voltammetry (AdSV) can be fashioned by embedding a suitable functional ligand in an electrically conductive porous matrix. Carbon graphite is often a choice of the conductive matrix because carbon paste electrodes afford low background currents, large potential domain, low costs, and ease of preparation and regeneration. The optimized detection procedure involves 
preconcentration (accumulation) of targeted metal ions on the electrode surface at open circuit, then desorption and cathodic electrolysis (deposition) of the preconcentrated species in an acidic medium, followed by a detection step using square wave anodic stripping voltammetry.

The voltammetric responses of carbon paste electrodes modified with thiol-SAMMS indicated that the electrodes are highly effective for simultaneous detection of $\mathrm{Pb}^{2+}$ and $\mathrm{Hg}^{2+}$ in aqueous solutions. The best electrode contained $20 \mathrm{wt} . \%$ of thiol-SAMMS and $80 \mathrm{wt} \%$ of carbon paste. The optimum operating conditions were a 2-5 minute preconcentration period in metal ion solution, followed by a 60 second cathodic electrolysis of the preconcentrated species in $0.2 \mathrm{M} \mathrm{HNO}_{3}$ and square wave anodic stripping voltammetric detection also in $0.2 \mathrm{M} \mathrm{HNO}_{3}$ solution. Under these conditions, the voltammetric responses (peak areas) after a 2-min preconcentration period were linear with respect to metal ion

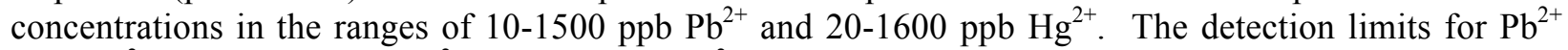
and $\mathrm{Hg}^{2+}$ were $0.5 \mathrm{ppb} \mathrm{Pb}^{2+}$ and $3 \mathrm{ppb} \mathrm{Hg}^{2+}$ after a 20 -minute preconcentration period. Effective simultaneous detections of $\mathrm{Cu}, \mathrm{Pb}, \mathrm{Cd}$, and $\mathrm{Hg}$ have also been achieved on carbon paste electrode modified with phosphonic acid SAMMS (Ac-Phos SAMMS).

In addition, the Ac_Phos_SAMMS modified carbon paste electrode has been demonstrated to be effective for detection of $\mathrm{U}(\mathrm{VI})$ in aqueous solution. U(VI) in $0.05 \mathrm{M}$ sodium acetate is accumulated on the electrode surface during an open circuit preconcentration step. Desorption and cathodic electrolysis at $-0.80 \mathrm{~V}$ was performed in $0.2 \mathrm{M} \mathrm{HNO}_{3}$. The voltammetric response is a linear function of preconcentration time. After a 5-minute preconcentration period, the uranium voltammetric response at $0.37 \mathrm{~V}$ is a function of $\mathrm{U}(\mathrm{VI})$ concentrations ranging from $25-500 \mathrm{ppb}$ in $0.05 \mathrm{M}$ acetate solution of $\mathrm{pH} 5$. As the preconcentration period is increased to 20 minutes, the uranium detection limit is improved significantly to $1 \mathrm{ppb}$.

\subsection{Electrochemical sensors based on microchip electrode modified with SAMMS thin-film.}

Another approach for the fabrication of SAMMS-based electrochemical sensor is by spin-coating the SAMMS thin-film on the surface of a microchip gold electrode (Figure 1).

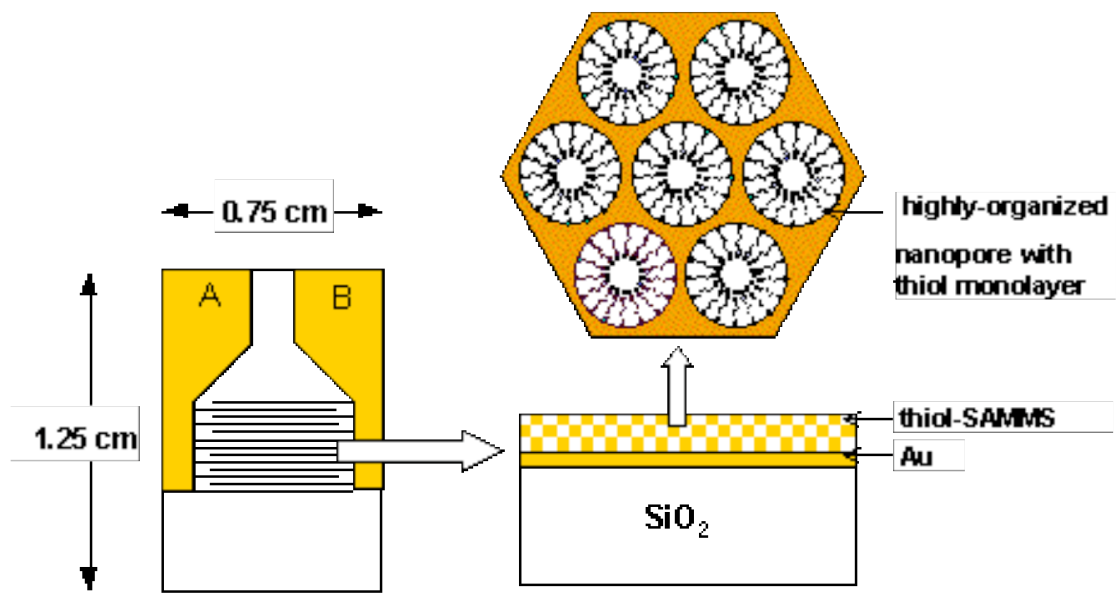

Figure 1. Electrochemical Sensor Based on Thiol-SAMMS Thin-Film Coated on Microchip Gold Electrode

Specifically, a mesoporous silica thin film was synthesized via surfactant-templating process by spincoating of the silica sol gel onto the surface of a gold electrode. Upon removal of the surfactant, a 3D hexagonal pore structured film with a primary pore size of $77 \AA$ was obtained, verified by TEM and XRD. Thiol (-SH) functional groups were subsequently immobilized onto the mesoporous silica thin film through self-assembly process. FTIR spectra indicate that thiol monolayer was successfully immobilized 
onto the surface of nanopores. The thiol-immobilized mesoporous silica (SH-SAMMS) thin film was used as the electrode sensing layer for the detection of lead(II) in aqueous solutions by employing square wave adsorptive stripping voltammetry technique. The SH-SAMMS thin film modified electrode utilizing the binding affinity of thiol ligands to lead(II) allowed the preconcentration step to be performed under an open circuit, with no additional electrolytes, and at the $\mathrm{pH}$ in which the silica thin film was stable (acidic to neutral $\mathrm{pH}$ ) .

From our preliminary study, the microchip gold electrode modified with SAMMS thin-film has demonstrated to be effective for lead(II) detection when coupled with adsorptive stripping voltammetry techniques similar to those performed on SAMMS-modified carbon paste electrodes. The lead(II) calibration curve was measured with the thiol-SAMMS coated microchip gold electrode, after a 5-minute preconcentration period. Sensitive and selective detection of $\mathrm{Pb}$ has been demonstrated. The advantage of microchip-based SAMMS Thin-film electrode is that the device is easy to be integrated with a microfluidic system.

The overall implications of these results provide an encouraging basis for the feasibility of development of microfluidic system integrated with SAMMS-based electrochemical sensor arrays. The immobilization of specific ligands on nanoporous thin films or carbon paste electrodes has demonstrated enhanced sensitivity and selectivity for the detection of metal ions of interest. This is a significant step toward the development of highly sensitive and selective microelectrochemical sensors for monitoring toxic transition metals and radionuclides in mixed wastes.

\section{Collaboration effort with NMSU}

The work performed at NMSU for FY 04 focused on various aspects of the development of microfluidics/electrochemical metal sensing system. The electrochemical sensors being investigated rely on the highly sensitive adsorptive stripping voltammetry (AdSV) technique to detect metal ions of interest to the DOE, particularly U(VI) and Cr(VI). Traditionally, AdSV measurements of U and Cr require the use of mercury electrodes which are not suitable for field deployment. Our initial goal was thus to replace these toxic mercury electrodes with 'environmentally-friendly' sensor materials. We have focused on bismuth-film electrodes, that were previously shown by our research group to be extremely useful alternatives to common mercury electrodes for stripping voltammetric measurements of heavy metals. Bismuth is an environmentally-friendly element, with very low toxicity, and a widespread pharmaceutical use. Our NMSU team has demonstrated recently the utility of bismuth electrodes for stripping detection of electrolytically deposited lead and cadmium.

In the present effort we have developed a sensitive adsorptive stripping voltammetric protocol at a bismuth-coated glassy-carbon electrode for trace measurements of chromium(VI) in the presence of diethylenetriammine pentaacetic acid (DTPA). The new protocol is based on accumulation of the CrDTPA complex at a preplated bismuth film electrode held at $-0.80 \mathrm{~V}$, followed a negatively-going squarewave voltammetric waveform. Factors influencing the stripping $\mathrm{Cr}$ response, including the film preparation, solution $\mathrm{pH}$, DTPA and nitrate concentrations, deposition potential, and deposition time, were systematically evaluated and optimized. The performance of bismuth-coated glassy-carbon electrode is comparable with that observed for analogous measurements at mercury film electrodes. Well defined $\mathrm{Cr}(\mathrm{VI})$ peaks were obtained for nanomolar levels of $\mathrm{Cr}$ in connection to short preconcentration times. A detection limit of $0.3 \mathrm{nM} \mathrm{Cr}(\mathrm{VI})$ was obtained using a 2 min accumulation. The bismuth-based electrodes showed good sensitivity, good reproducibility, and good selectivity. For example, a relative standard deviation of $5.1 \%$ was observed for 25 repetitive measurements of $20 \mathrm{nM} \mathrm{Cr}$. Applicability to selective measurements of $\mathrm{Cr}$ in real (river water) environmental samples was demonstrated. In the presences of $25 \mathrm{nM}$ (each) of $\mathrm{Pb}(\mathrm{II}), \mathrm{Cd}(\mathrm{II}), \mathrm{Zn}(\mathrm{II}), \mathrm{Cu}(\mathrm{II}), \mathrm{Fe}(\mathrm{III}), \mathrm{Ni}(\mathrm{II})$, and $\mathrm{Co}(\mathrm{II})$, the response for 5 $\mathrm{nM}$ chromium(VI) at our electrode was not affected. Also, large (20-fold) excess of hydrated $\mathrm{Cr}$ (III) did not affect the response of $5 \mathrm{nM} \mathrm{Cr}(\mathrm{VI})$, consistent with our previous studies that there was effective discrimination against hydrated $\mathrm{Cr}$ (III) species on bismuth-based electrodes. In addition to glassy-carbon substrates, we have used disposable thick-film (screen-printed) electrodes for supporting the bismuth film 
and found that the bismuth-coated on screen-printed electrodes offered a similar chromium detection as did the bismuth-coated glassy-carbon electrodes. The attractive feature of the new "mercury-free" chromium sensor holds a great promise for on-site environmental and industrial monitoring of chromium (VI). We are currently examining the use of bismuth film electrodes for sensitive adsorptive measurements of trace uranium.

Efficient microchip assays of real-world environmental samples will require the incorporation of a continuous sampling capability (from the external environment) or rapid sampling of multiple discrete samples. We have developed a rapid and reproducible sample introduction route into CE microchip devices based on alternatively placement of a sharp sample-inlet tip in the vials containing sample and buffer solutions (2). Such alternate placement of the inlet tip permits a volume-defined electrokinetic sample introduction. Such fast and simple sample introduction leads to highly reproducible signals with no observable carrying over of different analytes. Factors influencing the analytical performance of the new microchip interface were characterized and optimized. The attractive performance of the system was demonstrated in flow-injection and CE measurements. Employing of an 8-cm-long separation channel and a separation voltage of $4000 \mathrm{~V}$ offers high-throughput flow-injection assays of $100 \mathrm{samples} / \mathrm{hr}$ with a relative standard deviation of $3.7 \%(\mathrm{n}=100)$. Such ability to continuously introduce real samples into micrometer channels will make "Lab-on-a-chip" devices compatible with real-life environmental applications.

We have also developed a simple, user-friendly, and effective method for fabricating high-quality polymeric microchips based on an atmospheric pressure molding. The new fabrication route offers a substantial simplification of the entire fabrication process, through a judicious coupling of light-initiated polymerization of methylmethacrylate (PMMA) monomer solutions under ambient conditions with common photolithography and wet chemical etching techniques. This approach has significant advantages over conventional fabrication schemes of plastic devices; it does not require elevated pressures and temperatures (and subsequent cooling) or a complicated replication equipment. Variables of the fabrication process were assessed and optimized. High-quality devices with well-defined channels, injection-cross structures, and highly smoothed surfaces, were thus obtained. While the new approach was demonstrated in connection to PMMA microchips, it can be applied to other materials that undergo lightinitiated polymerization. The new fabrication approach brings significant simplification to the process of fabricating microfluidic devices and should lead to a widespread low-cost production of microchips.

In summary, our efforts during the first year of this project have focused on various aspects of the portable microfluidics/electrochemical metal sensing system. We have made a substantial progress, and introduced new and powerful protocols for stripping detections of heavy metal ions, uranium, and chromium.

\section{Planned Activities}

We will continue to optimize the coating technique for nanoporous silica thin-film. Monolayers of phosphonic acid functional group, which have proven to be highly efficient for selective actinides adsorption, will be incorporated as the thin film on the surface of nanoporous silica. Factors affecting the electrode performances (i.e., functional loading capacity, pore diameter, wall thickness, and thin-film thickness for the SAMMS thin-film electrode) and factors affecting the voltammetric detection process (i.e., preconcentration time, electrolysis time, stripping medium, $\mathrm{pH}$, etc.) will be investigated in details and the optimal operating parameters will be obtained. Interfacial chemistry and electrochemistry of metal species on surfaces of SAMMS-based electrodes will also be studied: this fundamental knowledge is required for predicting how the sensors will perform in the real wastes which consist of many interferences/ligands and are varied in $\mathrm{pH}$. The best electrodes for each specific waste constituent will be integrated onto the portable microfluidic platform. 


\section{Publications}

Lin Y, W Yantasee, and GE Fryxell. 2004. "Electrochemical Sensors Based on Functionalized Nanoporous Silica." In Dekker Encyclopedia of Nanoscience and Nanotechnology, ed. J. A. Schwarz, C. Contescu, K. Putye, pp. 1051-1062. Marcel Dekker, New York.

Fryxell GE, RS Addleman, SV Mattigod, Y Lin, TS Zemanian, H Wu, JC Birnbaum, J Liu, and X Feng. 2004. "Environmental and Sensing Applications of Molecular Self-Assembly ." In Dekker Encyclopedia of Nanoscience and Nanotechnology, ed. J. A. Schwarz, C. Contescu, K. Putye, pp. 1125-1135. Marcel Dekker, New York.

Kariuki NN, J Luo, L Han, MM Maye, L Moussa, M Patterson, Y Lin, MH Engelhard, and C Zhong. 2004. "Nanoparticle-Structured Ligand Framework as Electrode Interfaces." Electroanalysis 16(1-2):120126. (Special Issue: Nanotechnology)

Tu Y, Lin Y,Yantasee W, Ren ZF,2004. " Carbon nanotubes based nanoelectrode array: fabrication, evaluation, and application in voltammetric analysis." Electroanalysis, In press

Yantasee W, Y Lin, GE Fryxell, and BJ Busche. 2004. "Simultaneous Detection of Cadmium, Copper, and Lead using A Carbon Paste Electrode Modified with Carbamoylphosphonic Acid Self-Assembled Monolayer on Mesoporous Silica (SAMMS)." Analytica Chimica Acta 502(2):207-212.

L Lin, N S. Lawrence, S Thongngamdee, J Wang, Y Lin "Catalytic Adsorptive Stripping Determination of Trace Chromium (VI) at Bismuth Film Electrodes" Talanta, In press

Yantasee W, Y Lin, GE Fryxell, and Z Wang. 2004. "Carbon Paste Electrode Modified with Carbamoylphosphonic Acid Functionalized Mesoporous Silica: A New Mercury-Free Sensor for Uranium (VI) Detection." Electroanalysis. 16: 870-873

Y Lin, SK Fiskum, W Yantasee,H Wu, SV. Mattigod, GE. Fryxell, KN. Raymond, J Xu, Incorporation of Hydroxypyridinone (HOPO)Ligands into Self-Assembled Monolayers on Mesoporous Supports (SAMMS)for Selective Actinide Sequestration. Environ. Sci. Technol.(invited paper for special issue: Environ. \& Nanotechnology.) In Press.

Fryxell GE, H Wu, Y Lin, WJ Shaw, JC Birnbaum, JC Linehan, and Z Nie. 2004. "Lanthanide Selective Sorbents: Self-Assembled Monolayers on Mesoporous Supports (SAMMS) ." J. Materials Chemistry, In Press. 
GE. Fryxell, Y Lin, S Fiskum, JC. Birnbaum, H Wu. 2004. "Actinide Sequestration Using Functional Nanomaterials" Environ. Sci. Technol. (invited paper for special issue: Environ. \& Nanotechnology. Submitted.

Yantasee W, Y Lin, TS Zemanian, and GE Fryxell. 2003. "Voltammetric detection of lead (II) and mercury (II) using a carbon paste electrode modified with thiol self-assembled monolayer on mesoporous silica (SAMMS)." Analyst, 128(5):467-472.

Yantasee W, Y Lin, X Li, GE Fryxell, TS Zemanian, and VV Viswanathan. 2003. "Nanoengineered Electrochemical Sensor Based on Mesoporous Silica Thin-film Functionalized with Thiol-Terminated Monolayer." Analyst. 128, 899-904

Yantasee W, Y Lin, GE Fryxell, BJ Busche, and JC Birnbaum. 2003. "Removal of Heavy Metals from Aqueous Solution Using Novel Nanoengineered Sorbents: Self-Assembled Carbamoylphosphonic Acids on Mesoporous Silica." Separation Science and Technology. 38,1-17

"Fast and Simple Sample Introduction for Capillary-Electrophoresis Microsystems" G. Chen and J. Wang, Analyst, 129(2004)507.

\section{Presentations}

Fryxell GE, Y Lin, SV Mattigod, and H Wu. 2004. "Environmental Applications of Synthetic Nanoporous Ceramic Oxides." Presented by Glen E. Fryxell (Invited Speaker) at Clay Minerals Society, Richland, WA on June 23, 2004. PNNL-SA-41984, Pacific Northwest National Laboratory, Richland, WA.

Lin Y, Y Tu, F Lu, and Z Ren. 2004. "Carbon Nanotubes Based Nanoelectrode Arrays: Fabrication, Evaluation, and Sensing Applications." Presented by Yuehe Lin at Joint Regional Meeting of the 59th Northwest/18th Rocky Mtn ACS Regl Mtg 2004 , Logan, UT on June 7, 2004. PNWD-SA-6539, Battelle-Pacific Northwest Division, Richland, WA.

Lin Y. 2004. "Electrochemical Sensors and Biosensors Based on Nanostructured Materials." Presented by Yuehe Lin (Invited Speaker) at The 7th Asian Conference On Analytical Sciences, Hong Kong, on July 29, 2004. PNNL-SA-41668, Pacific Northwest National Laboratory, Richland, WA.

Lin Y, W Yantasee, GE Fryxell, X Li, and TS Zemanian. 2004. "Nanoengineered Electrochemical Sensors for Detection of Toxic Metals and Uranium." Presented by Yuehe Lin at The 227th ACS National Meeting, Anaheim, CA, March 28-April 1, 2004., Anaheim, CA on March 30, 2004. PNNLSA-40687, Pacific Northwest National Laboratory, Richland, WA. 